\title{
Hybrid optimal scheme for minimizing machining force and surface roughness in hard turning of AISI 52100 steel
}

\author{
P. Umamaheswarrao ${ }^{a *}$, D. Ranga Raju ${ }^{\text {b }}$, K.N.S. Suman ${ }^{c}$, B. Ravi Sankar ${ }^{d}$ \\ ${ }^{a *}$ Department of Mechanical Engineering, Bapatla Engineering College, Bapatala, A.P INDIA \\ ${ }^{b}$ Department of Mechanical Engineering, Srinivasa Institute of Engineering and Technology, Amalapuram, A.P INDIA \\ ${ }^{c}$ Department of Mechanical Engineering, College of Engineering Andhra University, A.P INDIA \\ ${ }^{d}$ Department of Mechanical Engineering, Bapatla Engineering College, Bapatala, A.P INDIA \\ "Corresponding Author: e-mail: maheshponugoti@gmail.com, Tel +91-9440871256
}

\begin{abstract}
In the present work hard turning of AISI 52100 steel has been performed using PCBN tools. The input parameters considered are cutting speed, feed, depth of cut, Nose radius and negative rake angle and the measured responses are machining force $\left(\mathrm{F}_{\mathrm{M}}\right)$ and surface roughness (Ra). Experiments are planned as per Center Composite rotatable Design (CCD) of Response Surface Methodology (RSM). Investigative analysis on the effect of input parameters on the response is carried out using main effects plot and response surface plots. Further, a multi-objective optimization is performed with RSM and Grey Relational Analysis (GRA) integrated with Principle Component Analysis (PCA). Results demonstrated that negative rake angle is the paramount factor affecting the response followed by feed, speed, depth of cut, and nose radius. The optimum cutting parameters obtained are cutting speed $1000 \mathrm{rpm}$, feed $0.02 \mathrm{~mm} / \mathrm{rev}$, depth of cut $0.4 \mathrm{~mm}$, Nose radius $1 \mathrm{~mm}$ and Negative rake angle 5
\end{abstract}

Keywords: Hard turning, AISI 52100 steel, Machining force, Surface roughness, Grey relational analysis.

DOI: http://dx.doi.org/10.4314/ijest.v11i3.3

\section{Introduction}

Turning of hardened steels with hardness greater than $45 \mathrm{HRC}$ is known as hard turning. Grinding is replaced by hard turning for hardened steel for finishing operations due to its process flexibility, high material removal rate, short cycle time and absence of coolant (König et al. 1984; Tönshoff et al. 2000). The differences between conventional machining vs hard turning was given away in Fig.1. (Dogra et al., 2010). Fabrication of complex parts with the help of hard turning leads to reduction of manufacturing cost by 30\% (Huang et al., 2007). Meddour et al. (2015) revealed that the force components were notably influenced by depth of cut followed by feed rate during AISI 52100 hard turning. Vrabel' et al. (2016) reported that surface roughness was highly affected by feed rate than cutting speed. Further, the feed rate was the most considerable factor for minimizing cutting force components. Ildikó et al. (2016) disclosed that surface roughness and cutting force components is influenced by cutting speed and feed in hard turning with coated ceramics. Azizi et al. (2016) observed that cutting forces increased as a function of work piece hardness and cutting time when turning was carried out with TiN coated ceramic inserts. Ouahid et al. (2017) noticed that feed rate has the major influence on surface quality and cutting force components majorly influenced by depth of cut.

Bartarya and Choudhury (2014) conducted the study with uncoated CBN tool and observed that feed and depth of cut were the most significant parameters affecting the forces. Surface roughness was highly influenced by depth of cut. In hard turning of AISI 52100 steel with PCBN tool feed rate has a significant influence on the surface finish while cutting speed and depth of cut had marginal effect (Gabriel et al., 2015), the tool geometry nose radius had a great influence (Ravi Sankar and Umamaheswarrao, 2017). Saurabh et al. (2018) optimized machining parameters during turning of hard porcelain using Taguchi and Response surface methodology. GRA coupled with PCA for multi-objective optimization of parameters was performed by Pradhan (2013), Vijian and Seshagiri Rao (2015), Umamaheswarrao et al. (2018) and gained wider success. 


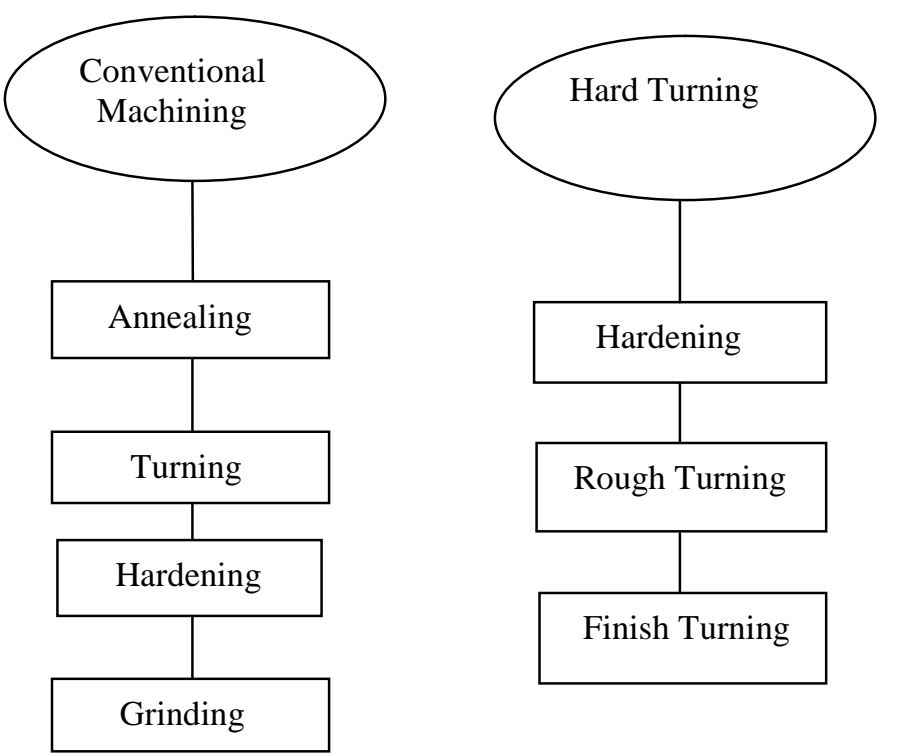

Figure 1. Distinction between conventional machining and hard turning (Dogra et al., 2010)

From the literature, it was elucidated that hard turning was the best alternative to grinding owing to its merits. Much emphasis was made on hard tuning of AISI 52100 steel by several researchers due to its applications in various parts of industry. The past studies made a large amount of interest to investigate the effect of cutting parameters on the responses. However, small insight was put on the investigations related to tool geometry such as nose radius, negative rake angle etc. Numerous authors adopted various optimization techniques for improving machining performance and integration of RSM, GRA and PCA was rarely deployed. Hence, the present study was aimed to conduct AISI 52100 steel hard turning using PCBN tools with cutting speed, feed rate and depth of cut as cutting conditions, nose radius and negative rake angle as tool parameters. Machining force and surface roughness were considered as responses. Further, multi response optimization was performed by integrating RSM, GRA and PCA for optimum cutting conditions.

\section{Experimental Details}

\subsection{Workpiece}

AISI 52100 steel is used as workpiece with a length of $500 \mathrm{~mm}$ and diameter of $48 \mathrm{~mm}$. Length of machining was $30 \mathrm{~mm}$ for each experimental run. Hardness of the workpiece used is $57 \mathrm{HRC}$.

Table 1. Factors and their levels

\begin{tabular}{|c|c|c|c|c|c|c|c|}
\hline S.No & Factors & Notation & \multicolumn{5}{|c|}{ Levels } \\
\hline & & & -2 & -1 & 0 & 1 & 2 \\
\hline 1 & Speed $(\mathrm{rpm})$ & $v$ & 200 & 400 & 600 & 800 & 1000 \\
\hline 2 & Feed $(\mathrm{mm} / \mathrm{rev})$ & $\mathrm{f}$ & 0.02 & 0.04 & 0.06 & 0.08 & 0.1 \\
\hline 3 & Depth of Cut $(\mathrm{mm})$ & $\mathrm{d}$ & 0.4 & 0.5 & 0.6 & 0.7 & 0.8 \\
\hline 4 & Nose radius $(\mathrm{mm})$ & $\mathrm{r}$ & 0.4 & 0.6 & 0.8 & 1 & 1.2 \\
\hline 5 & Negative rake angle $\left({ }^{\circ}\right)$ & $\alpha$ & -5 & -15 & -25 & -35 & -45 \\
\hline
\end{tabular}

\subsection{Cutting tool}

PCBN tool (Fig.2) with different nose radii i.e. 0.4, 0.6, 0.8, 1, $1.2 \mathrm{~mm}$ is deployed for experimentation with varied negative rake angles $-5,-15,-25,-35,-45$. ISO Geometric designations of the inserts are CNMG 120404, CNMG 120406, CNMG 120408, CNMG 120410 and CNMG 120412.

In dry condition experiments were carried out using on Kirloskar Turn master-35 type lathe. PSBNR2525 M12 type tool holder was used for mounting inserts. Experimental setup was shown in Fig.3. The initial cutting parameters were selected as cutting speed $200 \mathrm{rpm}$, feed $0.02 \mathrm{~mm} / \mathrm{rev}$, depth of cut $0.4 \mathrm{~mm}$, nose radius $0.4 \mathrm{~mm}$ and negative rake angle $5^{\circ}$. Factors and their levels are given away in Table 1. Kistler three-component measuring system (model 9257B) was used to measure cutting forces. The turned 
samples surface roughness was measured with Mitutoyo make Surface roughness tester (SJ-210). Experimental matrix with obtained responses is given in Table 2 .

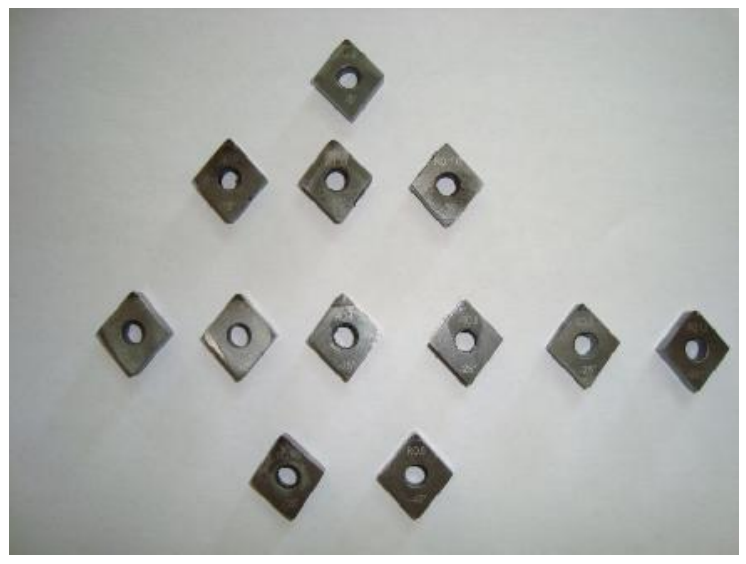

Figure 2. PCBN tools

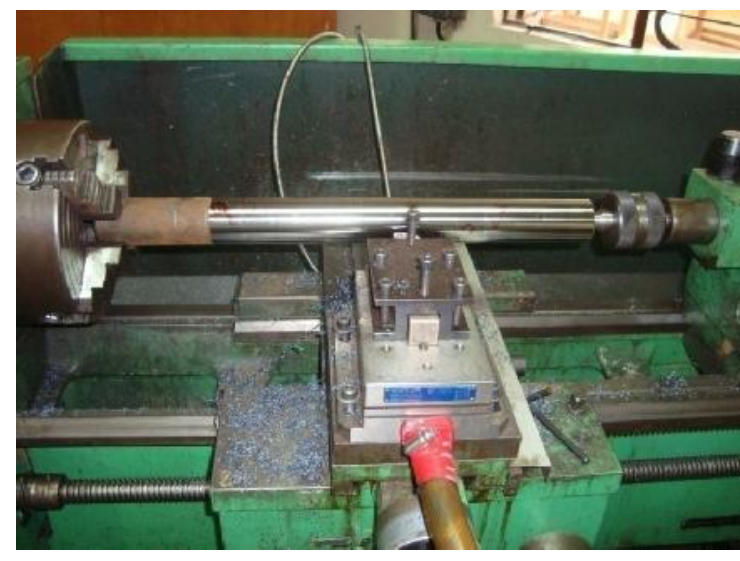

Figure 3. Experimental setup

\section{Methodology Adopted}

\subsection{Hybrid GRA-PCA}

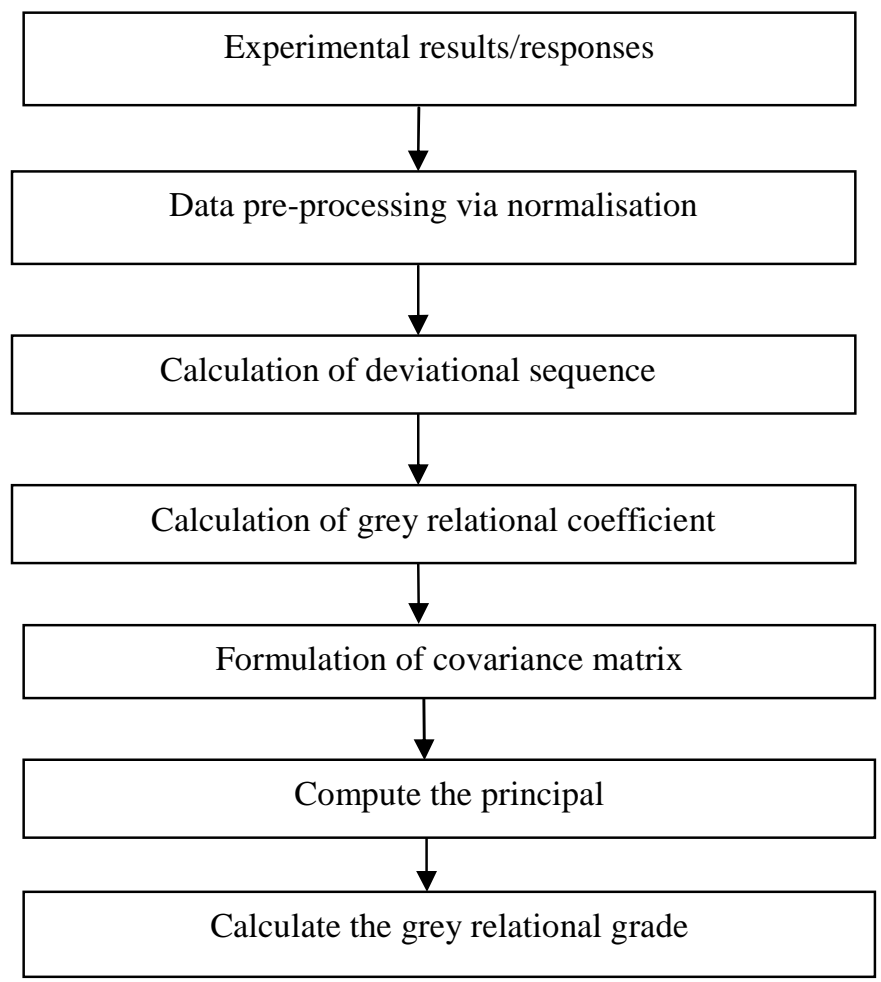

Figure 4. Steps in hybrid GRA-PCA

Optimum combination of various input parameters are determined by deploying GRA and PCA to obtain the best quality characteristics. (Wang et al., 2013; Hotelling H., 1993). Steps in hybrid GRA-PCA is given away in Fig.4. Normalized values and Deviational sequences are given away in Table 3. 
Table 2. Experimental matrix with responses

\begin{tabular}{|c|c|c|c|c|c|c|c|}
\hline $\begin{array}{l}\text { Exp. } \\
\text { No }\end{array}$ & $\begin{array}{c}v \\
(\mathrm{rpm})\end{array}$ & $\begin{array}{c}\mathrm{f} \\
(\mathrm{mm} / \mathrm{rev})\end{array}$ & $\begin{array}{c}\mathrm{d} \\
(\mathrm{mm})\end{array}$ & $\begin{array}{c}\mathrm{r} \\
(\mathrm{mm})\end{array}$ & $\begin{array}{c}\alpha \\
\left(^{\circ}\right)\end{array}$ & $\begin{array}{l}\mathrm{F}_{\mathrm{M}} \\
(\mathrm{N})\end{array}$ & $\begin{array}{c}\mathrm{Ra} \\
(\mu \mathrm{m})\end{array}$ \\
\hline 1 & 400 & 0.04 & 0.5 & 0.6 & 35 & 404.735 & 0.525 \\
\hline 2 & 800 & 0.04 & 0.5 & 0.6 & 15 & 233.475 & 0.465 \\
\hline 3 & 400 & 0.08 & 0.5 & 0.6 & 15 & 322.117 & 0.453 \\
\hline 4 & 800 & 0.08 & 0.5 & 0.6 & 35 & 473.03 & 0.545 \\
\hline 5 & 400 & 0.04 & 0.7 & 0.6 & 15 & 317.493 & 0.552 \\
\hline 6 & 800 & 0.04 & 0.7 & 0.6 & 35 & 376.384 & 0.507 \\
\hline 7 & 400 & 0.08 & 0.7 & 0.6 & 35 & 583.032 & 0.539 \\
\hline 8 & 800 & 0.08 & 0.7 & 0.6 & 15 & 380.407 & 0.471 \\
\hline 9 & 400 & 0.04 & 0.5 & 1 & 15 & 273.585 & 0.485 \\
\hline 10 & 800 & 0.04 & 0.5 & 1 & 35 & 425.463 & 0.401 \\
\hline 11 & 400 & 0.08 & 0.5 & 1 & 35 & 561.163 & 0.507 \\
\hline 12 & 800 & 0.08 & 0.5 & 1 & 15 & 350.276 & 0.502 \\
\hline 13 & 400 & 0.04 & 0.7 & 1 & 35 & 443.782 & 0.508 \\
\hline 14 & 800 & 0.04 & 0.7 & 1 & 15 & 323.621 & 0.408 \\
\hline 15 & 400 & 0.08 & 0.7 & 1 & 15 & 411.791 & 0.604 \\
\hline 16 & 800 & 0.08 & 0.7 & 1 & 35 & 523.367 & 0.498 \\
\hline 17 & 200 & 0.06 & 0.6 & 0.8 & 25 & 430.828 & 0.559 \\
\hline 18 & 1000 & 0.06 & 0.6 & 0.8 & 25 & 355.441 & 0.456 \\
\hline 19 & 600 & 0.02 & 0.6 & 0.8 & 25 & 309.595 & 0.468 \\
\hline 20 & 600 & 0.1 & 0.6 & 0.8 & 25 & 534.481 & 0.53 \\
\hline 21 & 600 & 0.06 & 0.4 & 0.8 & 25 & 344.431 & 0.45 \\
\hline 22 & 600 & 0.06 & 0.8 & 0.8 & 25 & 449.219 & 0.48 \\
\hline 23 & 600 & 0.06 & 0.6 & 0.4 & 25 & 359.396 & 0.514 \\
\hline 24 & 600 & 0.06 & 0.6 & 1.2 & 25 & 446.225 & 0.485 \\
\hline 25 & 600 & 0.06 & 0.6 & 0.8 & 5 & 279.954 & 0.484 \\
\hline 26 & 600 & 0.06 & 0.6 & 0.8 & 45 & 601.276 & 0.509 \\
\hline 27 & 600 & 0.06 & 0.6 & 0.8 & 25 & 358.525 & 0.507 \\
\hline 28 & 600 & 0.06 & 0.6 & 0.8 & 25 & 370.743 & 0.518 \\
\hline 29 & 600 & 0.06 & 0.6 & 0.8 & 25 & 378.525 & 0.52 \\
\hline 30 & 600 & 0.06 & 0.6 & 0.8 & 25 & 403.976 & 0.512 \\
\hline 31 & 600 & 0.06 & 0.6 & 0.8 & 25 & 380.24 & 0.488 \\
\hline 32 & 600 & 0.06 & 0.6 & 0.8 & 25 & 370.65 & 0.522 \\
\hline
\end{tabular}

The obtained Eigen values and Eigen vectors are shown in Table $4 \&$ Table 5. The GRC and GRG for the experimental runs are shown in Table 6. Fig.5 shows the variation of GRG with an experimental run. 
Table 3. Normalized values and Deviational sequences

\begin{tabular}{|c|c|c|c|c|}
\hline & \multicolumn{2}{|c|}{ Normalized Values } & \multicolumn{2}{|c|}{ Deviation Sequences } \\
\hline $\begin{array}{l}\text { Exp. } \\
\text { No }\end{array}$ & $\begin{array}{l}\text { Machining } \\
\text { force }\end{array}$ & Surface roughness & $\begin{array}{l}\text { Machining } \\
\text { force }\end{array}$ & Surface roughness \\
\hline 1 & 0.53436 & 0.389162 & 0.46563 & 0.610837 \\
\hline 2 & 1 & 0.684729 & 0 & 0.315270 \\
\hline 3 & 0.758994 & 0.743842 & 0.241005 & 0.256157 \\
\hline 4 & 0.348683 & 0.290640 & 0.651316 & 0.709359 \\
\hline 5 & 0.77156 & 0.256157 & 0.22843 & 0.743842 \\
\hline 6 & 0.611450 & 0.477832 & 0.388549 & 0.522167 \\
\hline 7 & 0.04960 & 0.320197 & 0.95039 & 0.679802 \\
\hline 8 & 0.600512 & 0.655172 & 0.399487 & 0.344827 \\
\hline 9 & 0.890946 & 0.586206 & 0.109053 & 0.413793 \\
\hline 10 & 0.478011 & 1 & 0.521988 & 0 \\
\hline 11 & 0.109061 & 0.477832 & 0.890938 & 0.522167 \\
\hline 12 & 0.682434 & 0.502463 & 0.317565 & 0.497536 \\
\hline 13 & 0.428204 & 0.472906 & 0.571795 & 0.527093 \\
\hline 14 & 0.754905 & 0.965517 & 0.245094 & 0.034482 \\
\hline 15 & 0.515183 & 0 & 0.484816 & 1 \\
\hline 16 & 0.211823 & 0.522167 & 0.788176 & 0.477832 \\
\hline 17 & 0.463424 & 0.221674 & 0.536575 & 0.778325 \\
\hline 18 & 0.668391 & 0.729064 & 0.331608 & 0.270935 \\
\hline 19 & 0.793040 & 0.669950 & 0.206959 & 0.330049 \\
\hline 20 & 0.181606 & 0.36453 & 0.818393 & 0.63546 \\
\hline 21 & 0.698325 & 0.75862 & 0.301674 & 0.24137 \\
\hline 22 & 0.413421 & 0.610837 & 0.586578 & 0.389162 \\
\hline 23 & 0.657638 & 0.443349 & 0.342361 & 0.556650 \\
\hline 24 & 0.421562 & 0.586206 & 0.578437 & 0.413793 \\
\hline 25 & 0.873630 & 0.591133 & 0.126369 & 0.408866 \\
\hline 26 & 0 & 0.467980 & 1 & 0.532019 \\
\hline 27 & 0.660006 & 0.477832 & 0.339993 & 0.522167 \\
\hline 28 & 0.626787 & 0.42364 & 0.373212 & 0.57635 \\
\hline 29 & 0.605629 & 0.413793 & 0.394370 & 0.586206 \\
\hline 30 & 0.536431 & 0.45320 & 0.463568 & 0.54679 \\
\hline 31 & 0.600966 & 0.571428 & 0.399033 & 0.428571 \\
\hline 32 & 0.627040 & 0.403940 & 0.372959 & 0.596059 \\
\hline
\end{tabular}

Table 4. Eigen values and explained variation for Principal components

\begin{tabular}{|c|c|c|}
\hline Principal component & Eigen value & Explained Variations (\%) \\
\hline First & 1.2406 & 64.03 \\
\hline Second & 0.6969 & 35.96 \\
\hline
\end{tabular}

Table 5. The Eigenvectors for principal components and contribution

\begin{tabular}{|c|c|c|c|}
\hline \multirow{2}{*}{ Responses } & \multicolumn{2}{|c|}{ Eigenvectors } & \multirow{2}{*}{ Contribution } \\
\cline { 2 - 3 } & $\begin{array}{c}\text { First principal } \\
\text { component }\end{array}$ & $\begin{array}{c}\text { Second principal } \\
\text { component }\end{array}$ & \\
\hline Machining force & 0.7071 & -0.7071 & 0.49999 \\
\hline Surface roughness & 0.7071 & 0.7071 & 0.49999 \\
\hline
\end{tabular}


Table 6. GRC, GRG and rank of the Machining force, Surface roughness

\begin{tabular}{|c|c|c|c|c|}
\hline \multirow{2}{*}{$\begin{array}{c}\text { Exp. } \\
\text { No }\end{array}$} & \multicolumn{2}{|c|}{ GRC } & \multirow[t]{2}{*}{ GRG } & \multirow[t]{2}{*}{ Rank } \\
\hline & Machining force & Surface roughness & & \\
\hline 1 & 0.5177955 & 0.450110865 & 0.483943 & 23 \\
\hline 2 & 1 & 0.613293051 & 0.80663 & 1 \\
\hline 3 & 0.674759 & 0.661237785 & 0.667985 & 6 \\
\hline 4 & 0.4342853 & 0.413441955 & 0.423855 & 28 \\
\hline 5 & 0.6864046 & 0.401980198 & 0.544182 & 13 \\
\hline 6 & 0.5627147 & 0.489156627 & 0.525925 & 16 \\
\hline 7 & 0.3447332 & 0.423799582 & 0.384259 & 32 \\
\hline 8 & 0.5558719 & 0.591836735 & 0.573843 & 10 \\
\hline 9 & 0.8209459 & 0.547169811 & 0.684044 & 4 \\
\hline 10 & 0.4892422 & 1 & 0.744606 & 3 \\
\hline 11 & 0.3594696 & 0.489156627 & 0.424305 & 27 \\
\hline 12 & 0.6115716 & 0.501234568 & 0.556392 & 11 \\
\hline 13 & 0.4665069 & 0.486810552 & 0.476649 & 24 \\
\hline 14 & 0.6710558 & 0.935483871 & 0.803254 & 2 \\
\hline 15 & 0.5077088 & 0.333333333 & 0.420513 & 29 \\
\hline 16 & 0.3881457 & 0.511335013 & 0.449731 & 25 \\
\hline 17 & 0.4823575 & 0.391136802 & 0.436738 & 26 \\
\hline .18 & 0.6012443 & 0.6485623 & 0.624891 & 9 \\
\hline 19 & 0.7072539 & 0.602373887 & 0.654801 & 7 \\
\hline 20 & 0.3792494 & 0.440347072 & 0.40979 & 30 \\
\hline 21 & 0.6236949 & 0.674418605 & 0.649044 & 8 \\
\hline 22 & 0.4601602 & 0.56232687 & 0.511233 & 19 \\
\hline 23 & 0.5935692 & 0.473193473 & 0.533371 & 15 \\
\hline 24 & 0.4636336 & 0.547169811 & 0.505392 & 21 \\
\hline 25 & 0.7982503 & 0.550135501 & 0.674179 & 5 \\
\hline 26 & 0.3333333 & 0.484486874 & 0.408902 & 31 \\
\hline 27 & 0.5952426 & 0.489156627 & 0.542189 & 14 \\
\hline 28 & 0.5725982 & 0.464530892 & 0.518554 & 17 \\
\hline 29 & 0.5590522 & 0.46031746 & 0.509675 & 20 \\
\hline 30 & 0.5189044 & 0.477647059 & 0.498266 & 22 \\
\hline 31 & 0.5561527 & 0.538461538 & 0.547296 & 12 \\
\hline 32 & 0.572764 & 0.456179775 & 0.514462 & 18 \\
\hline
\end{tabular}

Table 7. Mean response table for GRG

\begin{tabular}{|c|c|c|c|c|c|}
\hline Level & $v$ & $\mathrm{f}$ & $\mathrm{d}$ & $\mathrm{r}$ & $\alpha$ \\
\hline 1 & 0.436738 & $0.654801 *$ & $0.649044^{*}$ & 0.533371 & $0.674179 *$ \\
\hline 2 & 0.510735 & 0.633654 & 0.59897 & 0.551328 & 0.632105 \\
\hline 3 & 0.620178 & 0.533871 & 0.527036 & 0.535716 & 0.53255 \\
\hline 4 & 0.510735 & 0.48761 & 0.522295 & $0.569937 *$ & 0.489159 \\
\hline 5 & $0.624891 *$ & 0.40979 & 0.511233 & 0.505392 & 0.408902 \\
\hline Delta & 0.188153 & 0.245011 & 0.137811 & 0.064545 & 0.265277 \\
\hline Rank & 3 & 2 & 4 & 5 & 1 \\
\hline
\end{tabular}




\section{Results and Discussion}

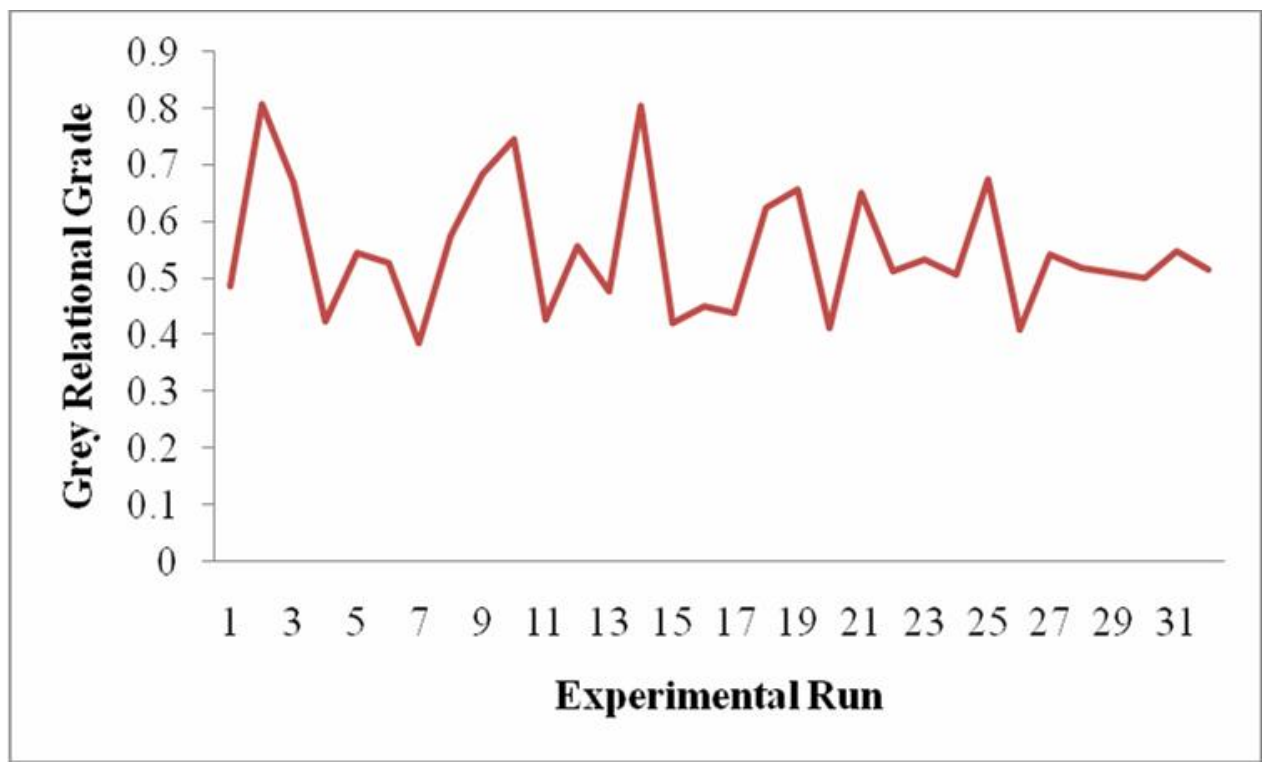

Figure 5. Experimental Run Vs GRG

The larger GRG indicates the better multiple-performance characteristics and therefore, the levels at which the largest average response was obtained was selected. In the response table (Table 7) negative rake angle has been assigned a rank 1 hence, it is the paramount parameter in controlling the response followed by feed, speed, depth of cut, and nose radius. From the ANOVA analysis, it is clear that negative rake angle contribution is highest (34.03\%) followed by feed (33.53\%), speed (17.55\%), depth of cut $(12.12 \%)$ and nose radius $(2.24 \%)$ as shown Table 8. Estimated regression coefficients for GRG are shown in Table 9.

Table 8. ANOVA for GRG

\begin{tabular}{|c|c|c|c|c|c|c|}
\hline Source & DF & SS & MS & F & P & \% Contribution \\
\hline Speed & 4 & 0.0634 & 0.0158 & 1.27 & 0.307 & 17.55 \\
\hline Feed & 4 & 0.1211 & 0.0303 & 2.92 & 0.040 & 33.53 \\
\hline Depth of cut & 4 & 0.0438 & 0.0109 & 0.83 & 0.520 & 12.12 \\
\hline Nose radius & 4 & 0.0081 & 0.0020 & 0.14 & 0.967 & 2.24 \\
\hline Negative rake angle & 4 & 0.1229 & 0.0307 & 2.98 & 0.037 & 34.03 \\
\hline Error & 11 & 0.0018 & 0.0004 & & & 0.49 \\
\hline
\end{tabular}

The regression coefficients are estimated for responses and the modeling is done considering $95 \%$ confidence level and hence those terms having $\mathrm{P}$ value $>0.05$ are insignificant. The adequacy of the developed model is judged by the $\mathrm{R}^{2}$ value and is $98.7 \%$ which is beyond $75 \%$ indicates the model is in good agreement. Quadratic equation for GRG after eliminating insignificant terms is shown below

$\mathrm{GRG}=0.519009+0.097888 * v-0.138198 * \mathrm{f}-0.074085 * \mathrm{~d}+0.007743 * \mathrm{r}-0.139510 * \alpha+0.069325 * \mathrm{~d} * \mathrm{~d}-0.146210 * v$

$* \mathrm{f}+0.063986 *{ }_{v} * \mathrm{~d}+0.074647 *{ }_{v} * \mathrm{r}-0.136719 * \mathrm{f} * \mathrm{r}+0.101436 * \mathrm{r} * \alpha$

From the main effect plot (Fig.6), it is observed that the optimistic grey relational grade can be achieved with Speed $=1000 \mathrm{rpm}$, feed $=0.02 \mathrm{~mm} / \mathrm{rev}$, depth of cut $=0.4 \mathrm{~mm}$, nose radius $=1 \mathrm{~mm}$, negative rake angle $=5^{\circ}$ respectively.

From the response plots (shown in Figure 7) it is evident that higher GRG was noticed at higher limits of speed, nose radius and negative rake angle and at lower limits of depth of cut and feed. Nose radius exhibited interaction with speed, feed and negative rake angle.

GRG for the obtained optimum combination of parameters was 0.98424 estimated from Eq. 2 and was $22.01 \%$ higher than highest GRG in Table 6, which indicates confirmation of optimality.

$$
\left.\gamma=\gamma_{m}+\sum_{i=1}^{q} \overline{\left(\gamma_{j}\right.}-\gamma_{m}\right)
$$


Table 9. Estimated Regression Coefficients for GRG

\begin{tabular}{|c|c|c|c|c|}
\hline Term & Coef & SE Coef & $\mathrm{T}$ & \\
\hline Constant & 0.519009 & 0.008703 & 59.633 & 0.000 \\
\hline$v$ & 0.097888 & 0.008908 & 10.989 & 0.000 \\
\hline $\mathrm{f}$ & -0.138198 & 0.008908 & -15.514 & 0.000 \\
\hline $\mathrm{d}$ & -0.074085 & 0.008908 & -8.317 & 0.000 \\
\hline $\mathrm{r}$ & 0.007743 & 0.008908 & 0.869 & 0.403 \\
\hline$\alpha$ & -0.139510 & 0.008908 & -15.661 & 0.000 \\
\hline$v * v$ & 0.020001 & 0.016115 & 1.241 & 0.240 \\
\hline $\mathrm{f} * \mathrm{f}$ & 0.021482 & 0.016115 & 1.333 & 0.209 \\
\hline$d * d$ & 0.069325 & 0.016115 & 4.302 & 0.001 \\
\hline $\mathrm{r} * \mathrm{r}$ & 0.008568 & 0.016115 & 0.532 & 0.606 \\
\hline$\alpha^{*} \alpha$ & 0.030727 & 0.016115 & 1.907 & 0.083 \\
\hline$v * \mathrm{f}$ & -0.146210 & 0.021820 & -6.701 & 0.000 \\
\hline$v * \mathrm{~d}$ & 0.063986 & 0.021820 & 2.932 & 0.014 \\
\hline$v^{*} \mathrm{r}$ & 0.074647 & 0.021820 & 3.421 & 0.006 \\
\hline$v * \alpha$ & -0.012108 & 0.021820 & -0.555 & 0.590 \\
\hline $\mathrm{f} * \mathrm{~d}$ & 0.031255 & 0.021820 & 1.432 & 0.180 \\
\hline$f^{*} \mathrm{r}$ & -0.136719 & 0.021820 & -6.266 & 0.000 \\
\hline$f^{*} \alpha$ & 0.017601 & 0.021820 & 0.807 & 0.437 \\
\hline $\mathrm{d} * \mathrm{r}$ & 0.023751 & 0.021820 & 1.088 & 0.300 \\
\hline $\mathrm{d}^{*} \alpha$ & 0.033278 & 0.021820 & 1.525 & 0.155 \\
\hline$r^{*} \alpha$ & 0.101436 & 0.021820 & 4.649 & 0.001 \\
\hline
\end{tabular}

\section{Main Effects Plot (data means) for GRG}

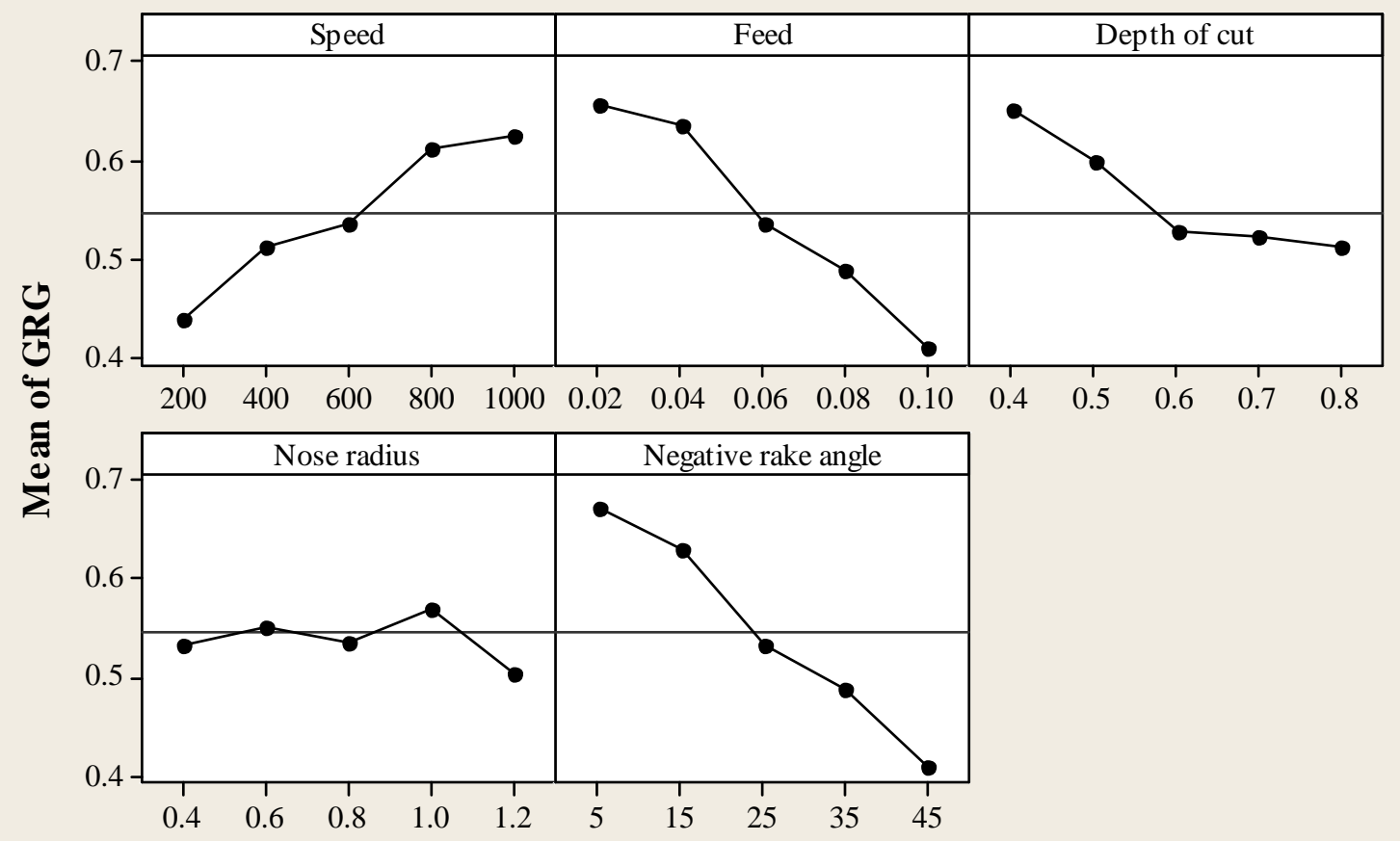

Figure 6. Main effects plot for GRG 


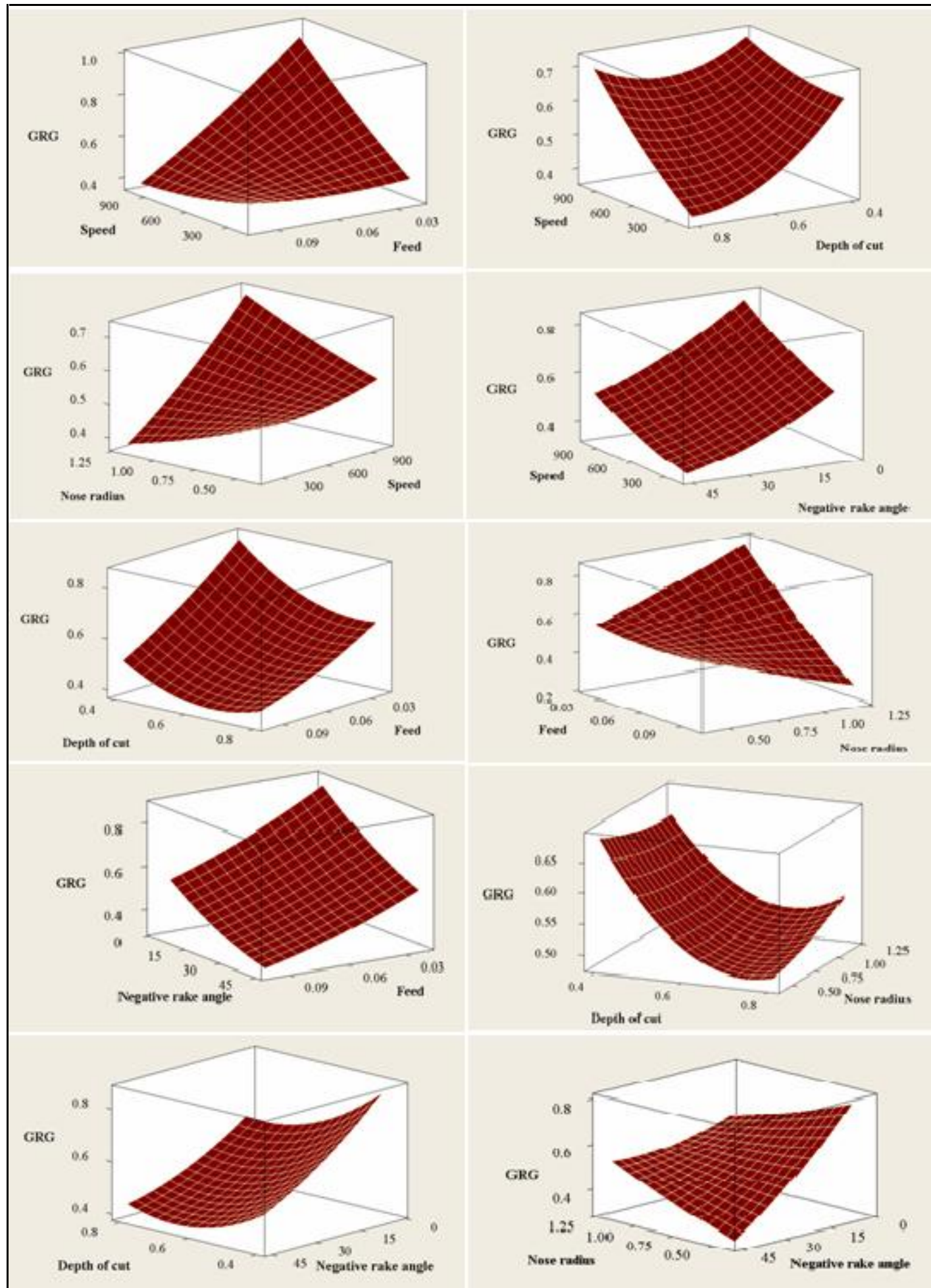

Figure 7. Response plots for GRG [Hold Values Speed 600; Feed 0.06; Depth of cut 0.6; Nose radius 0.8; Negative rake angle $\left.25^{\circ}\right]$

\section{Conclusions}

The optimization of parameters in hard turning of AISI 52100 steel was carried out with multiple performance characteristics. The experiments were conducted as per Center Composite Rotatable Design (CCD) of RSM and multi-objective optimization was performed using GRA coupled with PCA. 
- Optimum parametric settings and their levels were A5B1C1D4E1 i.e (Speed $=1000 \mathrm{rpm}$, feed $=0.02 \mathrm{~mm} / \mathrm{rev}$, depth of cut = $0.4 \mathrm{~mm}$, Nose radius $=1 \mathrm{~mm}$ and Negative rake angle $=5^{\circ}$ ).

- Responses were most significantly affected by negative rake angle followed by feed, speed, depth of cut, and nose radius.

- The interaction effect was found among speed and nose radius, nose radius and negative rake angle, feed and nose radius.

\section{Nomenclature}

$\begin{array}{ll}\text { RSM } & \text { Response Surface Method } \\ \text { GRG } & \text { Grey Relation Grade } \\ \text { GRA } & \text { Grey Relation Analysis } \\ \text { GRC } & \text { Grey Relation Coefficient } \\ \text { PCA } & \text { Principle Component Analysis } \\ \text { ANOVA } & \text { Analysis of Variance } \\ \text { CBN } & \text { cubic boron nitride } \\ \text { PCBN } & \text { Polycrystalline cubic boron nitride } \\ \gamma & \text { Predicted Grey relational grade } \\ \gamma_{m} & \text { Total mean of Grey relational grade } \\ \overline{\gamma_{j}} & \text { Mean of Grey relational grade at the optimal level }\end{array}$

\section{Acknowledgment}

Authors sincerely thank Karunya Institute of Technology and Sciences, Coimbatore, India for rendering their amenities and the technical support to accomplish this research work.

\section{References}

Azizi M.W., Belhadi S., Yallese M.A., Lagred A., Bouziane A. and Boulanouar L., 2016. Study of the machinability of Hardened 100Cr6 Bearing Steel With TiN coated Ceramic Inserts, Third International Conference on Energy, Materials, Applied Energetics and Pollution (ICEMAEP2016) October 30-31, Constantine, Algeria

Bartarya G. and Choudhury S.K., 2014. Influence of machining parameters on forces and surface roughness during finish hard turning of EN-31 steel, Proceedings of IMechE Part B: Journal of Engineering Manufacture, Vol. 228, No. 9, pp. 1068-1080.

Dogra M., Sharma V.S., Sachdeva A., Suri N.M., Singh J. and Dureja., 2010. Tool Wear, Chip Formation and Work piece Surface Issues in CBN Hard Turning: A Review, International Journal of Precision Engineering and Manufacturing, Vol. 11, No. 2, pp. 341-358.

Gabriel B., Danut S. and Adrian O., 2015. Influence of the cutting parameters on the surface roughness when machining hardened steel with ceramic and PCBN cutting tools, Advanced Engineering Forum, Vol. 13, pp. 19-22.

Hotelling H., 1993. Analysis of a complex of statistical variables into principal components, Journal of Educational Psychology, Vol. 24, pp. 417-441.

Huang O.Y., Chou Y.K. and Liang Y.S., 2007. CBN tool wear in hard turning: a survey on research progresses. International Journal of Advanced Manufacturing Technology, Vol. 35, pp. 443-453.

Ildikó M., Marek V., Jozef B. and Mária F., 2016. Modelling and Analysis of Relationship between Cutting Parameters Surface Roughness and Cutting Forces Using Response Surface Methodology when Hard Turning with Coated Ceramic Inserts, Key Engineering Materials, ISSN: 1662-9795, Vol. 686, pp. 19-26.

König W., Hochschule T., Komanduri R., Schenectady D. and Tönshoff H.K., 1984. Machining of hard materials. Ann CIRP, Vol.33, No. 2, pp. 417-427.

Meddour I., Yallese M. A., Khattabi R., Elbah M. and Boulanouar L., 2015. Investigation and modeling of cutting forces and surface roughness when hard turning of AISI 52100 steel with mixed ceramic tool: cutting conditions optimization, International Journal of Advanced Manufacturing Technology, Vol. 77, Issue. 5, pp. 1387-1399.

Ouahid K., Boulanouar L., Azizi M.W. and Yallese M.A., 2017. Effects of coating material and cutting parameters on the surface roughness and cutting forces in dry turning of AISI 52100 steel, Structural Engineering and Mechanics, Vol. 61, No. 4, pp. 519-526.

Pradhan M.K., 2013. Estimating the effect of process parameters on MRR, TWR and radial overcut of EDMed AISI D2 tool steel by RSM and GRA coupled with PCA. International Journal of Advanced Manufacturing Technology, Vol. 68, No.1-4, pp. 591-605.

Ravi Sankar B. and Umamaheswar rao P., 2017. Analysis of Forces during Hard Turning of AISI 52100 Steel Using Taguchi Method. Materials Today: Proceedings, Vol. 4, pp. 2114-2118. 
Saurabh A., Manoj kumar G., Dinesh kumar K. and Sharad A., 2018. Optimization of machining parameters of hard porcelain on a CNC machine by Taguchi-and RSM method, International Journal of Engineering, Science and Technology, Vol. 10, No. 1, pp. 13-22.

Tönshoff H.K., Arendt C. and Amor R.B., 2000. Cutting of hardened steel. Ann CIRP, Vol. 49, No. 2, pp. 547-566.

Umamaheswarrao P., Ranga Raju, D., Naga Sai Suman, K. and Ravi Sankar B., 2018. Multi objective optimization of process parameters for hard turning of AISI 52100 steel using Hybrid GRA-PCA, Procedia Computer Science, Vol.133, pp.703-710.

Vijayan D. and Seshagiri Rao V., 2015. Parametric optimization of age hardenable aluminum alloys using TGRA coupled with PCA, Applied Mechanica and Materials, Vol. 813-814, pp. 613-619.

Vrabel' M., Maňková I., Kovač P., Beňo J., Franková M. and Pal’o M., 2016. Analysis and optimization of Hard Turning Process using $\mathrm{Al}_{2} \mathrm{O}_{3} /$ Ticn Ceramic TiN PVD Coated Insert With Regard to Surface Roughness and Cutting Force Components, Journal of Production Engineering, Vol. 19, No. 1, pp. 22-26.

Wang P., Meng P., Zhai J.Y. and Zhou-Quan Zhu Z-Q., 2013. A hybrid method using experiment design and grey relational analysis for multiple criteria decision making problems, Knowledge-Based Systems, Vol. 53, pp. 100-107.

\section{Biographical notes}

P. Umamaheswarrao and B. Ravi Sankar are of the Department of Mechanical Engineering, Bapatla Engineering College, Bapatala, India.

D. Ranga Raju is of the Department of Mechanical Engineering, Srinivasa Institute of Engineering and Technology, Amalapuram, A.P India

K.N.S. Suman is of the ${ }^{c}$ Department of Mechanical Engineering, College of Engineering Andhra University, A.P India

Received May 2018

Accepted April 2019

Final acceptance in revised form April 2019 